\title{
Host Bacterial Interactions in Periodontal Disease - An Overview
}

\section{IJCRR}

Section: Healthcare

Sci. Journal Impact

Factor: 5.385 (2017)

ICV: 71.54 (2015)

\section{Sujeetha ${ }^{1}$, Anitha Logaranjani ${ }^{2}$, Vijayalakshmi R. ${ }^{2}$, Rajapriya ${ }^{3}$, Jaideep Mahendra ${ }^{4}$}

'MDS, PG student, Department of Periodontology, Faculty of Dentistry, Meenakshi Academy of Higher Education and Research, Chennai, India; ${ }^{2}$ MDS, Reader, Department of Periodontology, Faculty of Dentistry, Meenakshi Academy of Higher Education and Research, Chennai, India; ${ }^{3} \mathrm{MDS}$, Senior lecturer, Department of Periodontology, Faculty of Dentistry, Meenakshi Academy of Higher Education and Research, Chennai, India; ${ }^{4} \mathrm{MDS}$, PhD Post Doc (USA), Director of Post graduate studies, Professor Department of Periodontology, Faculty of Dentistry, Meenakshi Academy of Higher Education and Research, Chennai, India.

\section{ABSTRACT}

There are several microbiological and immunological aspects involved in the interactions between bacteria and the host during the initiation and progression of periodontal disease. Periodontal therapy lacks the diagnostic capability to tailor predictable therapy for the individual. An understanding of the various mechanisms involved will enable us to plan better therapeutic approach that aims in the eradication of the disease process. In future, we can improve the host's ability and thereby prevent the colonization of harmful periodontal pathogens. This review article provides the current state of the art in regard to bacterial host cell interactions that take place during periodontal disease pathogenesis

Key Words: Adhesins, Evasion, Immunity, Invasion, P. gingivalis

\section{INTRODUCTION}

Periodontitis is a complex multifactorial infectious disease of the supporting structures of the teeth characterized by destruction of alveolar bone and connective tissue. The primary etiologic agents for causing peritonitis are the specific periodontopathic bacteria and their virulence factors. The collective interaction of these etiologic agents and the host defence mechanism responses along with the environmental factors play an important role in the onset and progression of the disease.

This review article provides the current state of the art in regard to bacterial host cell interactions that take place during periodontal disease pathogenesis.

\section{Microbiological aspects of the microbial host interactions}

Dental plaque forms an integral part of the microbiological aspect of periodontal disease ${ }^{1}$. The process of plaque formation can be divided into three major phases i. The formation of the pellicle on the tooth surface

ii. Initial adhesion and attachment of the bacteria

iii. Colonization and plaque maturation

These processes have been extensively described in the microbiological aspects of the host microbial interactions as:

A. Bacterial colonization and survival in the periodontal region

B. Microbial mechanisms of host tissue damage

\section{A. Bacterial colonization and survival in the periodontal region}

Periodontal disease progression is dependent on the simultaneous recurrence of a number of factors. The bacterial species which enhance infection must be present in the local environment or it should not inhibit the pathogen's activity. The host must be susceptible both locally and systemically. The environment also must be promotive to the expression of virulence factors by the pathogen. The virulence factors are the properties of the microorganism that enable it to cause disease. They enhance the microbial activity on periodontal tissues. They can be broadly categorised into two groups ${ }^{1}$ :

\section{Corresponding Author:}

Dr. Vijayalakshmi R., MDS, Reader, Department of Periodontology, Faculty of Dentistry, Meenakshi Academy of Higher Education and Research, Chennai, India; Email: srvijayalakshmi@rediffmail.com

ISSN: 2231-2196 (Print)

Received: 22.03 .2019
ISSN: $0975-5241$ (Online)

Revised: 25.04 .2019
Accepted: 14.05 .2019 
1. Factors that enable a bacterial species to colonize and invade host tissues and

2. Factors that enable a bacterial species to cause tissue damage directly or indirectly.

\section{Adherence}

Adherence represents a virulence factor for periodontal pathogens. Infections are generally initiated by bacteria to a wide variety of biotic and abiotic host surfaces ${ }^{2}$. Many pathogenic bacteria have developed incredibly large and diverse array of adhesive molecules, called adhesins, on their surfaces. The adhesins interact with a variety of host components and are responsible for recognizing and binding to specific host cell receptor moieties.

Adhesins can be subdivided into two major classes ${ }^{3}$ :

1. Fimbrialadhesins

2. Non-fimbrialadhesins

\section{Fimbrialadhesins}

In the bacterial pathogenesis or infection, adherence is an essential step required for colonizing a new host. Fimbrial adhesins are appendages or cell surface components of bacteria which facilitates bacterial adhesion to other cells or to inanimate surfaces. They are a type of virulence factor. In gram-negative bacteria, the fimbriae function as adhesins. In many cases, the actual adhesin is a minor subunit protein at the tip of the fimbriae. Ingram-positive bacteria, the specific adhesion is a protein or polysaccharide surface layer.

Fimbrial adhesins in gram negative bacteria are classified into five major classes based on their biosynthetic pathway:
i. Chaperone - usher pili (CU pili)
ii. Curli
iii. Type IV pili
iv. Type III secretion pili
v. Type IV secretion pili

i. Chaperone-usher pili (CU pili)

Pili and fimbriae are thin, filamentous, proteinaceous surface appendages that protrude from the surface of many different bacterial species and are prominent on gram negative bacteria where they are anchored within the outer membrane. In $\mathrm{CU}$ pili, a periplasmic chaperone and outer membrane assembly platform known as "usher" are strictly required for the pilus assembly.

\section{ii. Curli}

Curli are thin aggregative fimbriae that were identified as a new type of fimbrialadhesin expressed on the outer surfaces of some Enterobacteriae.

iii. Type IV pili

Type IV pili are extruded across the outer membrane and form long and flexible surface appendages.

\section{iv. Type III secretion system}

It is a complex assembly that spans the inner membrane, periplasmic space, outer membrane and cellular membrane of the host. Several species of gram negative bacteria like the Salmonella, Shigella, Yersenia, Escherichia and Pseudomonas use this to inject bacterial proteins as a way to translocate substrates directly into the cytoplasm of the host cell where they exert a broad range of virulence functions.

\section{v. Type IV secretion system pili}

This not only translocates effectors into eukaryotic cells, but also mediates the transfer of a single stranded DNA molecule, resulting in genetic colonization of the host. Gene acquisition is an important adaptive mechanism that enables pathogens to respond to a changing environment during invasion of the host and T4SS pili are thought to be able to establish stable and specific contacts between cells before substrate transfer.

\section{Non fimbrial adhesins:}

These adhesins recognise many different elements on host cell surfaces, including components of the extracellular matrix such as collagen, laminin, elastin, proteoglycans, hyaluronan, vitronectin, fibrinogen and fibronectin.

Types of non fimbrial adhesins:

i. Auto transporter adhesins

ii. Outer membrane adhesins

iii. Secreted adhesins

iv. Those associated with biofilm formation

\section{i. Autotransporter adhesins}

They constitute a family of outer membrane/secreted proteins used for translocation to the surface of the bacterium.

\section{ii. Outer membrane adhesins}

These outer membrane proteins contain a beta-barrel structure and function as a membrane anchor for adhesins.

\section{iii. Type 1 secretion system adhesin}

It is a translocator contained in gram negative bacteria which allows secretion of proteins of various sizes and functions from the cytoplasm to the extracellular medium in a single step.

\section{iv. Adhesins associated with biofilm formation}

Although exopolysaccharides are important matrices to develop biofilm, a large family of adhesins, termed biofilm associated proteins, also play a leading role in the formation of microbial biofilms. 


\section{Adhesins of periodontal bacteria}

Adherence of bacteria to mucosal and tooth surfaces, as well as bacterial coaggregation, are essential steps for colonization with various oral bacterial species ${ }^{4}$.

\section{Fimbrial adhesins}

- Porphyromonas gingivalis expresses two distinct fimbriae-molecules on its cell surfaces: one is composed of a subunit protein encoded by the fimA gene and it is called the long, or major fimbriae, while the other consists of subunit minor fimbrial protein encoded by the mfal gene and it is called the short, minor, or mfa fimbriae.

- Aggregatibacter actinomycetemcomitans has both fimbrial and non-fimbrial adhesins on its surface. The bacterium possesses long bundles of pili.

- Prevotella intermedia has four morphologically distinct types of fimbriae and are designated as types A, $\mathrm{B}, \mathrm{C}$ and $\mathrm{D}$ based on their size.

- Fusobacterium nucleatum has Fusobacterium adhesion A, which is a novel fimbrial adhesion protein. It exists in two forms, namely the non-secreted form and the secreted mature form.

- In oral actinomyces species, A. viscosus expresses two distinct types of fimbriae (Type 1 and 2) which are involved in adherence to host tissues and inter bacterial coaggregation. Other actinomyces species lack fimbriae and their related genes.

\section{Non fimbrial adhesins}

- $\quad$ P. gingivalis has gingipain complex. The gingipains or extracellular cysteine proteinases are the product of three genes: two that code for arginine-specific genes (RgpA and RgpB) and one that code for lysine-specific proteinase gene (Kgp). Gingipains are involved in the virulence potential of $\mathrm{P}$. gingivalis. It influences the binding of the bacterium to the host tissues5.

- Hemin-binding protein (HBP) 35, may also be an essential protein for bacterial survival in evasion from environmental stress in Porphyromonas gingivalis. It binds hemin and expresses haemagglutination activity. HBP 35 is involved in multiple P. gingivalis binding to erythrocytes and host epithelial gingival cells. The virulence of $\mathrm{P}$. gingivalis may either directly or indirectly be affected by this protein. HBP35 is also involved with other bacteria6.

- The adherence of Treponemadenticola includes the number of components in the outer membrane which are the chymotrypsin-like protease, (also named dentilisin), major outer sheath protein, oligopeptidebinding protein ortholog, and a leucine-rich repeat protein.

- The non fimbrial adhesins of Tannerella forsythia is BspA, surface associated protein, encoded by the
bspA gene and S-layer proteins, outside the outer membrane of the organism.

- Non-fimbrial adhesins of Aggregatibacter actinomycetemcomitans has been identified on the surface of A. a, including three auto-transporter adhesins, Aae, ApiA, extracellular matrix protein adhesion $\mathrm{A}$ and various polysaccharides.

- In Fusobacterium nucleatum, two distinct types of adherence have been found and they are classified based on their inhibition mode using either D-galactose or L-arginine. Adherence to gram positive early colonizing species is associated with arginine inhibiting interactions whereas adherence to predominantly gram-negative late colonizing bacteria is associated with galactose-inhibiting interactions.

\section{Bacterial evasion of host defense mechanism}

To survive in the periodontal environment, bacteria must neutralize or evade the host defence mechanisms involved in bacterial clearance and killing. The host tissue invasion occurs by

1) The initial interaction with epithelial cells and

2) Through various bacterial entry mechanisms.

\section{1) Initial interaction with epithelial cells}

Interactions between bacteria and their surrounding epithelium are critical factors in bacterial infections. Adherence to epithelial cells is important for colonization. Besides serving as a physical barrier, the epithelium also functions as a sensor for the presence of bacteria. The direct physical contact between bacteria and the mucosal surface triggers the expression of a variety of immune response mediators from the epithelial cells.

\section{2) Bacterial entry mechanism}

Traverse of the plasma membrane by small molecules, such as ions and sugars, is easily mediated through various transmembrane channels and pumps in the bilayer but the transport of macromolecules through the plasma membrane requires endocytosis ${ }^{7}$. In endocytosis, membrane domain invaginate and are then pinched off from the inner side of the plasma membrane and transported within the cell. Most invasive bacteria utilise endocytosis to become engulfed by the host cells.

\section{Entry mechanism of various periodontal bacteria}

\section{i) P. gingivalis}

Entry mechanism of P. gingivalis exploits cellular endocytosis to enter a cell in a trigger mechanism like manner. It is considered to be mediated by the interaction between bacterial fimbriae and $\alpha 5 \beta 1$ integrin. P. gingivalis releases outer membrane vesicles in an extracellular manner, after which the bacteria retain the full range of outer membrane constituents. Intracellular P. gingivalis is localised in various cellular 
compartments which is specific to the type of the host cell. P. gingivalis is engulfed by endocytic vacuoles for entry, it must exit from endocytic vacuoles to localise in the cytoplasm. Following internalization, P. gingivalis may be able to replicate inside the cell. Some intracellular P. gingivalis are able to exit from the primarily infected host cell into the intercellular space and can enter new host cells, which enables futher penetration into host cells in a trans-cellular manner.

\section{ii) Aggregatibacter actinomycetemcomitans}

This bacterium can exploit cellular endocytosis to enter cells in a trigger mechanism like manner similar to P. gingivalis. The entry process is initiated by direct interactions between bacterial adhesins and cellular receptors. The bacteria are captured by ruffled pseudopodia and they are probably internalized via an endocytic pathway by an actin dependent mechanism. Those that are captured by endocytic compartments are suggested to destroy the compartment membranes, based on the secretion of phospholipase $\mathrm{C}$ and exit to the cytoplasm. Then it reportedly undergoes rapid multiplication and subsequently propagates in an intercellular manner. The bacteria residing in the cytoplasm can spread intracellularly to adjacent epithelial cells via intercellular protrusions. The direct cell-to-cell spreading allows bacteria to disseminate in infected tissues. Some strains were found to be internalized through actin-independent, receptor-mediated endocytosis.

\section{iii) Other bacteria}

- The "S- layer" isolated from Tannerella forsythia is shown to mediate haemagglutination, adhesion or invasion of epithelial cells. This layer is composed of two glycoproteins of molecular mass 200 and 210 $\mathrm{kDa}$.

- The entry mechanism of P. intermedia is because of its fimbriae. The type $\mathrm{C}$ fimbriae, present in $\mathrm{P}$. intermedia enables it to bind to human buccal epithelial cells more avidly than the other strains.

- Entry of Fusobacterium nucleatum into human gingival epithelial cells in-vitro was accompanied by an increased secretion of interleukin- 8 from the epithelial cells.

\section{B. Microbial mechanisms of host tissue damage}

Tissue damage is mainly caused by microbial virulence factors. Virulence factors are specific to each pathogenic bacteria and include sustenins, endotoxins, adhesins, invasins, bacteriocins, leukotoxin, Fc-binding proteins, immunosuppressive proteins, cytotoxins, collagenase, extracellular membranous vesicles, inhibitors of polymorphonuclear leukocytes function, proteases, fibronectin, laminin, fibrinogen, dentilisin and various fimbriae.

\section{Immunologic aspects of the microbial host interaction}

Innate immunity and adaptive immunity are two fundamental aspects of the immune system response to invading microbes $^{8}$. When there is a bacterial infection, in periodontal pathogenesis the role of immune system involves the following: firstly the innate factors such as a compliment, resident leukocytes and especially the mast cells play an important role in signalling endothelium thus initiating the inflammation; then the acute inflammatory cells protect the local tissues by controlling the periodontal microbiota within the gingival crevice and junctional epithelium; finally the chronic inflammatory cells, macrophages and lymphocytes protect the entire host from within the subjacent connective tissues and do all that is necessary to prevent a local infection from becoming systemic and life threatening, including the sacrifice of local tissues.

\section{The innate defence system}

Gingival crevice is the first region of the periodontium that comes into contact with microorganism that attempts to attach and colonize the area. Gingival epithelium provides a physical barrier to infection and has an active role in the innate host defence. The innate mechanisms which serve to prevent such microbial colonisation include, the mechanical washing effect of saliva and GCF and the detrimental effect of these fluid constituents on the bacterial growth.

The cells of the epithelium can respond to the bacteria by producing antimicrobial peptides including beta defensin and the release of interleukin-8 and interleukin-13. The polymorphonuclear leukocytes appear to play a key role in the maintenance of periodontal health, as molecular defects in PMN's, with a variety of functional consequences can result in accelerated periodontitis.

Important component of innate immune response is the toll like receptors. When microorganisms enter the tissue after penetrating the epithelial barrier, they are encountered by tissue macrophages, mast cells and immature dendritic cells. Apoptotic particles generated by normal tissue turnover and particles that are indicative of infection must be distinguished by these cells. The molecules mainly responsible for making this pivotal distinction are those of the family of Toll-like receptors9.

Members of the toll-like receptor family are responsible for the recognition of pathogen associated molecular patterns expressed by a wide spectrum of infectious agents. The signalling pathway of toll-like receptor consists of the MyD88dependent pathway and the MyD88-independent pathway.

\section{The adaptive defence system}

The adaptive response utilizes strategies of recognition, memory and binding to support the effector systems in the 
elimination of challenging elements. Parts of the adaptive response in periodontitis are: the nature of the lymphocyte type; antigen recognition by the toll-like receptor; cytokine profiles of $\mathrm{T}$ helper cells and autoimmune reactions that may influence the adaptive response in periodontitis.

The neutrophils reach the site of host bacterial interaction because of increased vascular permeability and engorgement of blood vessels produced by the inflammatory changes that occur due to the induction of cytokines by host cells as a result of the endotoxins produced by bacteria ${ }^{10}$.

The antigen presentation is one of the major component of acquired immune response where the recognition of the pathogen is done by the molecules called as the major histocompatibility complex. They are the central molecules that participate in the interaction during antigen presentation by antigen presenting cells to $\mathrm{T}$ cells.

Role of complement system in periodontal disease progression is important. The complement system can be activated by immune complexes and immunologic molecules like endotoxins. It occurs by three mechanisms, including the classical pathway, alternate pathway and the lectin pathway.

\section{Cell mediated immune response in periodontal diseases}

T-lymphocyte response to antigenic challenges is called the cell mediated immune response. T-lymphocytes can be functionally divided into CD4+ cells and CD8+ cells by the type of the antigen receptors. Helper T cells can be differentiated into T helper 1 and T helper 2 cells. They are distinguished by the cytokines they produce and respond to and are involved in different immune responses.

\section{Role of regulatory $\mathbf{T}$ cells in periodontal dis- eases}

These cells are different from Th1 or Th2 cells and play an important role in the cell mediated immune response. Three distinct regulatory $\mathrm{T}$ cells have been described including the naturally occurring Treg cells, CD4+ and CD 25+ T cells.

\section{Role of Th17 cells in periodontal disease}

A distinct type of helper T-cell lineage is Th17. It produces several proinflammatory cytokines including the cytokine interleukin-17 and hence the name. It is thought to play an important role in the pathogenesis of cell mediated tissue damage caused by autoimmunity or immune responses against microbial infection.

\section{Role of B cells}

They may be T-cell dependent or independent B-cell response, but B-cell response is actually regulated by T-cells. T-cell independent response occurs by cross-linking of the IgM by antigen receptor on the B-cell, responding with $\operatorname{IgM}$ synthesis in the absence of T-cell help. T-cell dependent activation of B-cells occurs when a pathogen is ingested by an antigen presenting cell such as a macrophage or dendritic cell. The pathogens proteins are then digested to peptides and attached to a class II MHC protein. This complex is then moved to the outside of the cell membrane. The antigen presenting cell then interacts with helper $T$ cell. This activation leads to the secretion of cytokines by helper T-cells which causes B-cell proliferation and maturation. Activated B-cells subsequently produce antibodies which assist in division of the pathogen ${ }^{11}$.

\section{DISCUSSION}

Precision medicine, as viewed by the National Institute of health, is disease treatment and prevention that accounts for individual variability. In general, periodontal therapy lacks diagnostic capability to tailor predictable therapy for the individual. Initial non-surgical therapy for chronic periodontitis consists of broad base reduction of ecological factors through scaling and root planning to control inflammation.

The initial periodontal therapy is generally successful in improving clinical measures of disease but assumes that all patients will respond in the same way. Furthermore, the clinical measures of periodontal disease status, including probing pocket depth, bleeding on probing and calculated measures of loss of attachment reflect the macroscopic history of disease progression rather than a more precise measure of real time changes of inflammation or advancing periodontitis ${ }^{12}$. Substantial work has been undertaken to identify risk factors or markers for disease progression.

Strategies to improve the host's ability by preventing bacterial colonization or eliminating the important microbial species can be employed in the future. For example, attention has been focused on eliciting immune responses to adhesins and proteases of Porphyromonas gingivalis. Alternatively preformed antigen and antibodies could be produced and applied to dental surfaces to prevent attachment and bacterial colonization.

In-vitro and in-vivo preclinical studies using NSAID's has shown the extensive ability of the drug to reduce prostanoid production by inhibiting the cyclooxygenases. The use of bone sparing drugs that inhibit alveolar bone resorption is another field in host modulation therapy ${ }^{13}$.

Inhibition of signal transduction pathways would be expected to abolish both cell activation by cytokines or other stimuli and the production of pro-inflammatory cytokines. Therapeutic strategies have been directed towards many of these major signalling pathways. The efficacy of anti-cytokine therapy in patients with inflammatory bone diseases is proof that blocking the effects of a cytokine can slow a disease 
process. The main advantages of pharmacological inhibitors are ease of administration and low cost compared with biotherapies or gene therapy. Osteoprotegerin is expressed by the osteoblastic cells and it acts as a decoy receptor. It binds to RANKL and inhibits osteoclast development. Reduction of the bacteria and their metabolic by products through periodontal therapy also results in a decrease in both interleukin-1 and tumor necrosis factor-alpha which is significantly elevated in diseased periodontal sites when compared with healthy or inactive sites and can result in bone resorption.

A variety of treatment strategies have been developed to target the host response to periodontal infection. Matrix metalloproteinase inhibitors, such as low dose formulations of doxycycline have been used in combination with scaling and root planning or surgical therapy. Other therapeutic strategies being explored are aimed at inhibiting signal transduction pathways involved in inflammation. Using these novel strategies, inflammatory mediators, including proinflammatory cytokines, matrix metalloproteinases and others would be inhibited at the level of cell signalling pathways required for transcription factor activation which is necessary for inflammatory gene expression or mRNA stability. These therapies may provide the next wave of disease specific chemotherapeutics to manage chronic periodontitis.

\section{CONCLUSION}

Recent investigations have revealed that not all strains of a specific microbial species are equal in their capacity to cause disease and not all hosts are equal in their susceptibility to disease. The challenge for the future is to be able to better identify the more virulent bacterial strains and the more susceptible host. In this manner it may be possible to predict accurately the individuals at risk for future disease and to develop more effective strategies to prevent the onset and progression of periodontitis. A new perspective has been emphasized about the influence of periodontal health on the overall health of the individual. In addition this new data automatically move bacteria mediated periodontal diseases more into the mainstream of medicine, a fact which is likely to have profound implication on our profession.

\section{ACKNOWLEDGEMENT}

Authors acknowledge the immense help received from the scholars whose articles are cited and included in references of this manuscript. The authors are also grateful to authors / editors / publishers of all those articles, journals and books from where the literature for this article has been reviewed and discussed.

\section{Source of Funding: Nil}

Conflict of interest: There was no conflict of interest

\section{REFERENCES}

1. Socransky SS and Haffajee AD. The bacterial etiology of destructive periodontal disease: current concepts. J Periodontol 1992;63(4):322-331.

2. Pizarro-Cerda $\mathrm{J}$ and Cossart P. Bacterial adhesion and entry into host cells. Cell 2006;124(4):715-727.

3. Soto GE, Hultgren SJ. Bacterial adhesins: common themes and variations in architecture and assembly. J Bacteriol 1999;181(4):1059-1071.

4. Darveau RP, Tanner A, Page RC. The microbial challenge in periodontitis. Periodontol 2000 1997;14:12-32.

5. Chen T, Nakayama K, Belliveau LL, Duncan MJ. Porphyromonas gingivalis gingipains and adhesion to epithelial cells. Infect Immun 2001;69(5):3048-3056.

6. Hiratsuka K, Hayakawa M, Kiyama-Kishikawa M, Sasaki Y, Hirai T, Abiko Y. Role of the hemin-binding protein 35 (HBP35) of Porphyromonas gingivalis in coaggregation. Microb Pathog 2008;44(4):320-328.

7. Conner SD, Schmid SL. Regulated portals of entry into the cell. Nature 2003;422(6927):37-44.

8. Azuma M. Fundamental mechanisms of host immune responses to infection. J Periodontal Res 2006;41(5):361-373.

9. Botos I, Segal DM, Davies DR. The structural biology of Tolllike receptors. Structure 2011;19(4):447-459.

10. Nathan C. Neutrophils and immunity: challenges and opportunities. Nat Rev Immunol 2006;6(3):173-182.

11. Carpenter AB, Sully EC, Ranney RR, Bick PH. T-cell regulation of polyclonal $\mathrm{B}$ cell activation induced by extracts of oral bacteria associated with periodontal diseases. Infect Immun 1984;43(1):326-336.

12. Hung HC, Douglass CW. Meta-analysis of the effect of scaling and root planning, surgical treatment and antibiotic therapies on periodontal probing depth and attachment loss. J Clin Periodontol 2002;29(11):975-986.

13. Shimizu N, Ozawa Y, Yamaguchi M, Goseki T, Ohzeki K, Abiko Y. Induction of COX-2 expression by mechanical tension force in human periodontal ligament cells. J Periodontol 1998;69(6):670-677. 